\title{
A Smart Metering Simulation in LABVIEW
}

\author{
Abdelmadjid RECIOUI", Hassiba DJEMAI and Farah BOUCENNA \\ Smart Grid Team, Laboratory of Signals and Systems, Institute of Electrical and Electronic \\ Engineering, University M'hamed Bougara of Boumerdes \\ Boulevard de l'indépendance, 35000, Boumerdes, Algeria \\ a_recioui@univ-boumerdes.dz
}

\begin{abstract}
The smart grid delivers electricity from producers to consumers using two-way digital technology and considers the involvement of the user. The implementation of smart grids should start at the consumer side using smart meters, control devices and a communication link. Smart metering is the bridge between smart buildings and smart grids. It is viewed as a crucial factor for an efficient functioning of the internal electricity market and a successful implementation of the renewable energy and security of supply. The replacement of the currently used electromechanical meters with smart digital meters along with domestic load controllers is identified for better energy conservation at the consumer side. These devices can be remotely read and are able to measure consumption multiple times per day which is beneficial for a good demand response, customer feedback and energy management. The purpose of the present paper is to illustrate how smart meters operate and how their implementation can improve the current grid
\end{abstract}

Keywords: Smart Grids, Smart Meters, Load Control, LABVIEW.

\section{INTRODUCTION}

Climate change, awareness of energy efficiency, new trends in electricity markets, the obsolescence of the actual electricity model, the urgent need to decarbonize electricity supply and swap aging resources and to make effective application of swiftly evolving information and communication technologies [4], and the gradual conversion of consumers to prosumer profiles are the main agents of progressive change in electricity systems towards the Smart Grid paradigm. The introduction of multiple distributed generation and storage resources, with a strong involvement of renewable energies, exposes the necessity of advanced metering or Smart Metering systems, able to manage and control those distributed resources [1].

Electricity networks are undergoing significant transformation. Clean, small, distributed energy and demand-side resources are challenging the traditional axiom of electricity from large, remote

Cite this article as:

Abdelmadjid RECIOUI, Hassiba DJEMAI and Farah BOUCENNA, "A Smart Metering Simulation in LABVIEW", Algerian Journal of Signals and Systems, Vol. 4, Issue 2, December 2019.pp: 89100. power generation facilities delivered over extensive transmission and distribution (T\&D) infrastructure to consumers.

This transformation is the result of a number of diverse and disruptive technology innovations. These innovations are changing the design of the electricity network, the flow of electricity in the system, and are driving utilities to forge a complex set of new relationships with stakeholders (e.g., end users, energy services companies, generators).

The backbone of this transformation is a modern electricity distribution system, a smart grid. A modern electricity distribution system is more complex, has greater redundancy, and allows for greater choice over the manner in which users generate, deliver, and consume electricity. A Smart Grid is a concept for transforming an electric power grid by using advanced communication, automated controls and other forms of information technology. It integrates new innovative tools and technologies from generation, transmission, and all the way to consumer appliances and equipment. [2].

The smart grid can be viewed as a superposition of communication networks on the electric grids. Hence, it can improve efficiency, reliability, safety, 
and security of electricity supply to the customers, with a seamless integration of renewable and alternative energy sources, through automated control and modern communications technologies where various components of the electric grid are linked together via two-way communications and power flows to provide interoperability among them. Thus, consumers can not only draw power but also supply surplus power to the grid using smart meters that enable monitoring and measuring of these bidirectional flows [3].

Smart metering has been recognized as a major part of the smart grid system, where it is built with smart meters, control devices and a communication link. The smart meter is the key element of this system which is the combination of all energy metering and intelligence, Smart meters are capable of communicating with each other and executing command signals remotely and locally. They provide good solutions in overcoming the problems that are faced with the old grid system.

Smart meters are embedded with different technologies and services. Therefore, implementation of smart meters appears to be multi-standard and incompatible but the meter developers ought to include the consideration of basic abilities such as:

1. Remote provision of metering data and related information to the utility

2. Two-way communications between the meter and the utility

3. Remote operation for disabling and enabling supply

4. Provision of information to home and networks

5. Automatic processing, transfer, management and utilisation of metering data

6. Security tampers detections and remote configurations [4].

With the increase in consumption of energy and population, there is a grave need to conserve energy in every way possible. The inability to access and control the appliances from remote locations is one of the major reasons for energy loss [5].

Home automation is building mechanization for a home, called a smart home. It includes the control and computerization of lighting, warming, (for example, brilliant indoor regulators), ventilation, aerating and cooling (HVAC), and security, and also home machines, for example, washer/dryers, stoves or fridges/cooler. And it does not only refer to reducing human efforts but also energy efficiency and time saving. Wi-Fi is regularly utilized for remote checking and control [6].

This paper proposes a design of a smart energy metering system. We will simulate a real time scenario which is done by changing the load profile. Furthermore, we will cover the general meaning of a direct load control and try to put it in our program as well. Finally, we will deal with the control center or Data Management System (DMS) which is in charge of receiving and storing the metering data for processing purposes.

\section{THE SMART METER}

The Smart meters are the first step in the building of a smart consumer interface. They have the capability to measure the bidirectional energy flow and communicate with each other to execute command signals remotely and locally. Smart meters have much functionality that need to be exploited in coordination of the system grid to produce real benefits (e.g. demand response).

Integration of unpredictable renewable power sources into the Grid is leading to the development of wide area control algorithms and smart grid. Whereas Smart meters are combined with modern communication technologies thus enabling cost-effective remote meter reading communication protocols and standers are needed to endure interoperability between different metering and communication technologies, the incorporation of modern telecommunication technologies has established a reliable communication link all over the smart grid system making it easy to monitor and control.

\section{A. Smart Meters:}

Smart meters are different from electronic meters because of their additional functionalities and features. Apart from electricity measurements and automatic meter reading (AMR), they allow two-way communication between the meter and the base station. Load profiling, prepayment, remote disconnection and reconnection, power outage notification, tamper detection, and multi-tariffing are also possible with smart meters. Therefore, AMR system is 
introduced by combining the communication infrastructure to electronic meters. Meanwhile more features and functions are added to AMR system. Ultimately Advanced Metering Infrastructure has been developed to today's technology with two-way communication and data management system. Fig. 1 shows the evolution of smart meter technology from electronic meters to AMI [7].

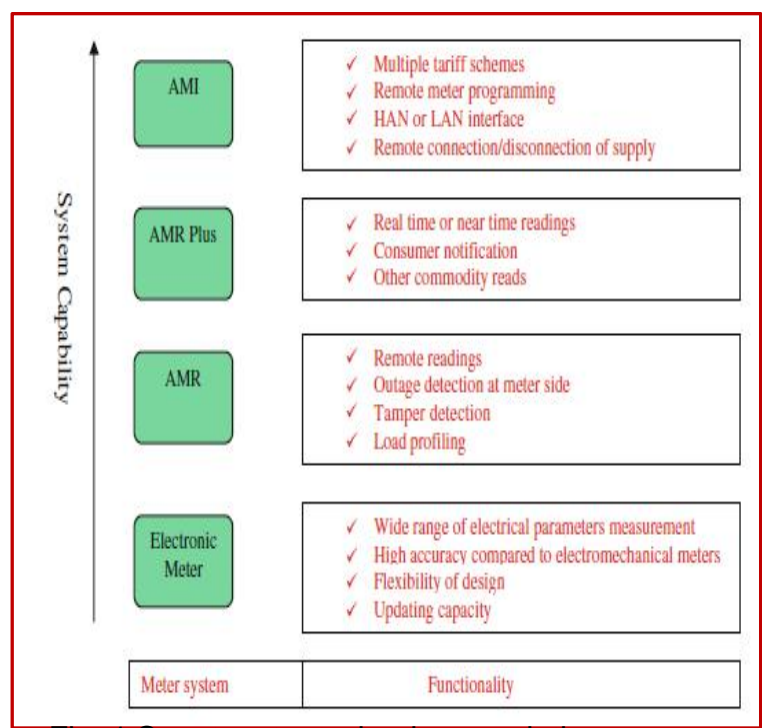

Fig. 1 Smart meter technology evolution

\section{B. The Hardware structure of a Smart Meter}

Figure 2 shows the functional bock diagram of a smart meter. It includes signal acquisition, signal conditioning, Analogue to Digital Conversation (ADC), computation and communication.

Smart meters use voltage and current sensors to get the input signals. Signal conditioning, $A D C$, and computations are done inside the micro controller unit (MCU). Additional hardware components are required for other operations like communication, time and date measurements, and data backup and storage. A smart meter is typically composed of following hardware components:

- Voltage and current sensing unit

- Power supply

- Energy measurement unit (metering IC)

- Microcontroller

- Real time cock

- Communicating system [7].

Figure 1-4 shows the modern hardware structure of smart meter.

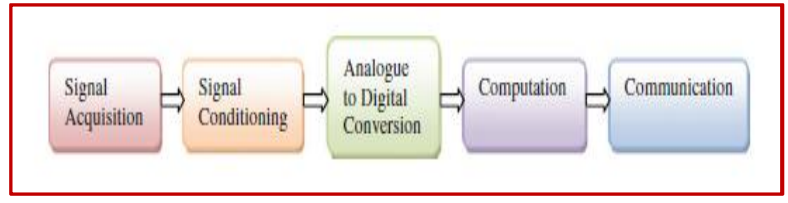

Fig. 2 Functional bock diagram of a smart meter.

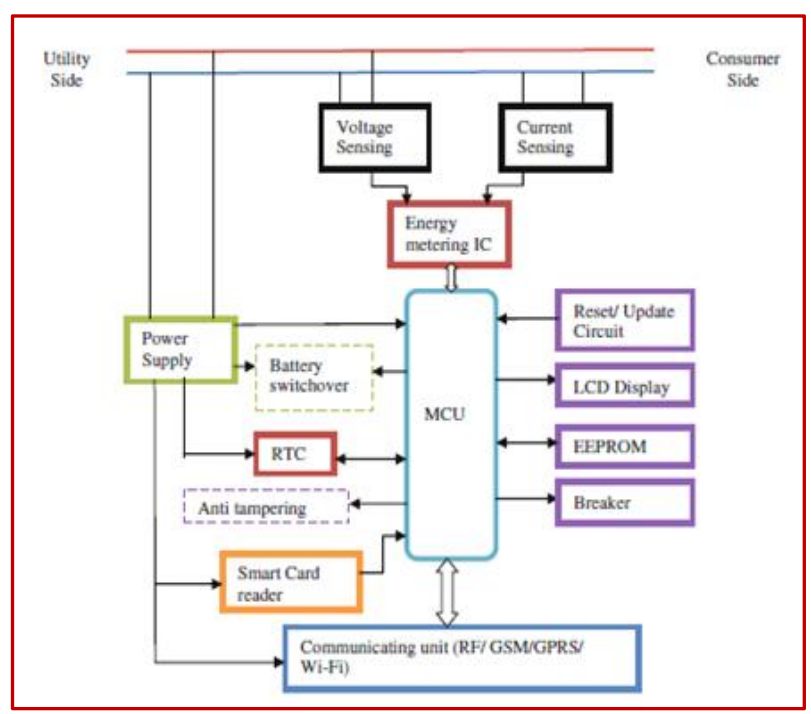

Fig. 3 Hardware structure of a modern smart meter.

C. Energy Measurement unit:

Signal conditioning, ADC, and computation are done inside the energy measurement unit. Energy measurement unit could be a standard energy measurement chip or the system MCU itself. Modern energy measurement chips have digital signal processor (DSP) to perform signal conditioning, ADC and energy calculations. These chips can be found as single phase energy measurement chips or multi phase energy measurement chips. They provide active, reactive, and apparent energy information as data or frequency (pulse) output. RMS voltage measurement, RMS current measurement, frequency, temperature measurement, tampering detection, power management, THD, line SAG detection and communication are also possible in some of them [7].

\section{a. Microcontroller:}

All functions inside the smart meter are performed by the MCU. It is considered as the core of the meter. It controls the following functions:

- Communication with the energy measurement chip

- Calculations based on the data received 
- Display electrical parameters, tariff and cost of electricity

- Smartcard reading

- Tamper detection

- Data management with EEPROM

- Communication with other communication devices

- Power management.

Smart meters are normally designed with a LCD. Therefore the consumer is updated with tariff and power outages. Sometimes alarm signals are generated to warn the consumer of higher tariffs and higher demands. Some meters use stepper motor counters rather than a LCD to display the energy consumptions. Those functions are also handled by the external MCU. Some smart meters consist with a single MCU which does all tasks including the energy measurement and routine arithmetic operations. In this case multi-tasking or high degree of parallelism is needed. In other words several operations must be performed simultaneously to the same data sets [7].

\section{b- Communicating System:}

The system that consists of smart meters, communication gateway, intelligent control, and data management is known as AMI. Several communication protocols are used in AMI. AMI can consist with a HAN (Home Area Network), a Neighborhood Area Network (NAN) and a WAN. Smart meters are the key elements in AMI which need to communicate with domestic appliances, other type of meters (typically water and gas meters), neighboring smart meters, and the energy supplier. HAN is used to establish a communication link between the smart meter and the smart appliances, other meters, inhome display, and the micro generation unit. HAN provides centralized energy management, services, and facilities. The communication protocol can be a wired or wireless media. Zig-bee, Z-wave, WI-Fi, and PLC are widely used protocols in HANs. PLC might be a cost effective approach for a HAN but it has many drawbacks due to its robustness. Zig-Bee communication has been recognized as a cost effective, less complexity, low power, and reliable media to handle a HAN].

A NAN is used to transfer the data between neighboring smart meters. It facilitates diagnostic messages, firmware upgrades, and real-time messages. Zig-bee communication protocol is widely used in
NAN due to high speed of data transferring and low cost.

Some smart meters are connected to a remote server through a WAN. They might not be connected to a NAN and data are directly transferred to the server using the wireless media. The communication is established between the meter and the server through a data concentrator for billing purposes, indication of power outages, remote disablement and enablement of supply, security tamper detections, and remote configurations. GSM, GPRS, 3G, and WiMax communication technologies can be used to connect the meter to the WAN. GSM provides wider coverage than other media. However, it will be costly in the long run [7].

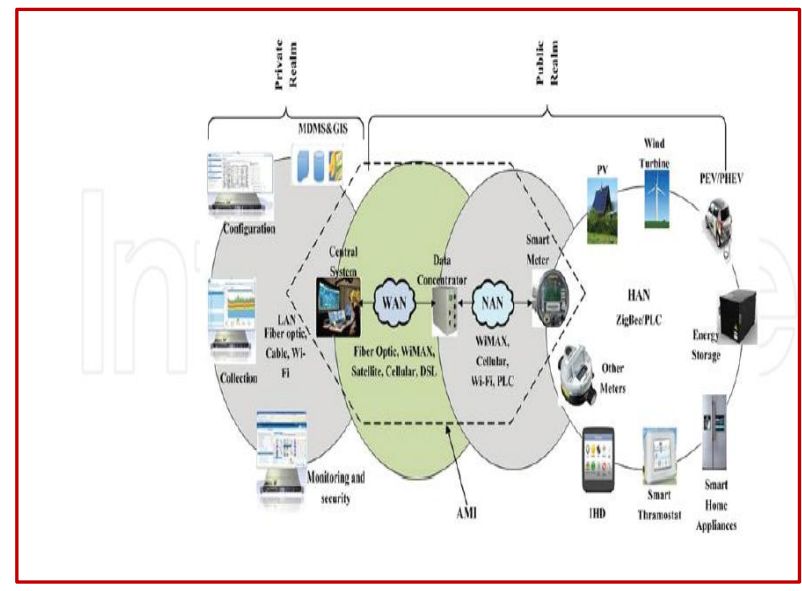

Fig. 4 AMl overview Characteristics.

\section{LABVIEW SIMULATION OF SMART METERING}

Smart meters are an advanced form of meters. They differ from electronic meters because of added functionalities. Apart from electricity measurements and automatic meter reading (AMR), they allow two-way communication between the meter and the utility.

This section presents the simulated smart metering system. It includes its design and its way of measuring the energy based on bidirectional capabilities including the photovoltaic solar panels system. At the end, we will simulate a real time scenario which can be done with the help of a load profile. Furthermore, we will cover the general meaning of a direct load control and try to put it in our program as well. Finally, we consider the control center or Data Management System (DMS) which is in charge of receiving 
and storing the metering data for processing purposes.

\section{A. Smart Metering System Simulation Using LABVIEW:}

a- Smart Meter Design:

The presentation of the smart meter design is divided into two sections:

$>\quad$ Customer energy consumption.

$>$ Photovoltaic panels Energy production.

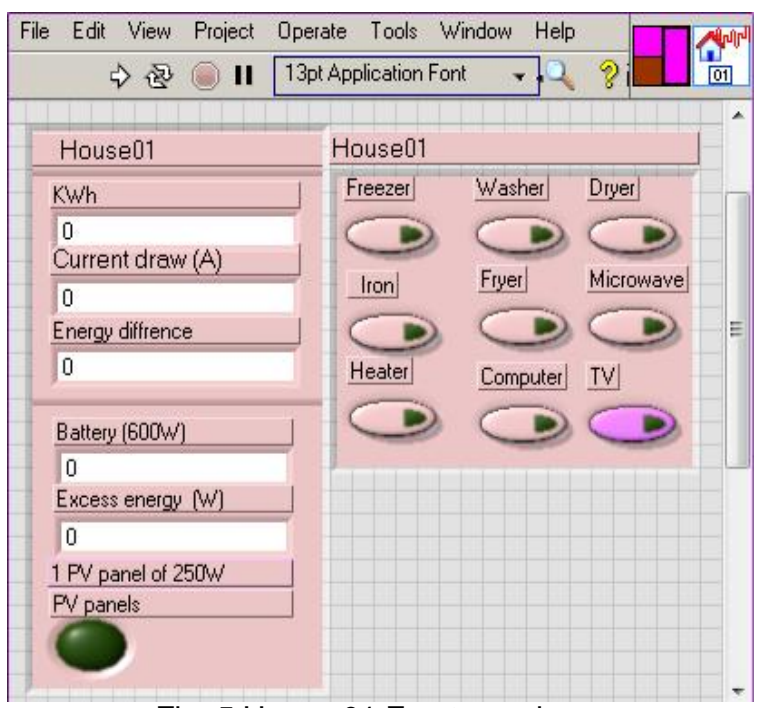

Fig. 5 House 01 Front panel.

Figure 5 shows the front panel of house 01

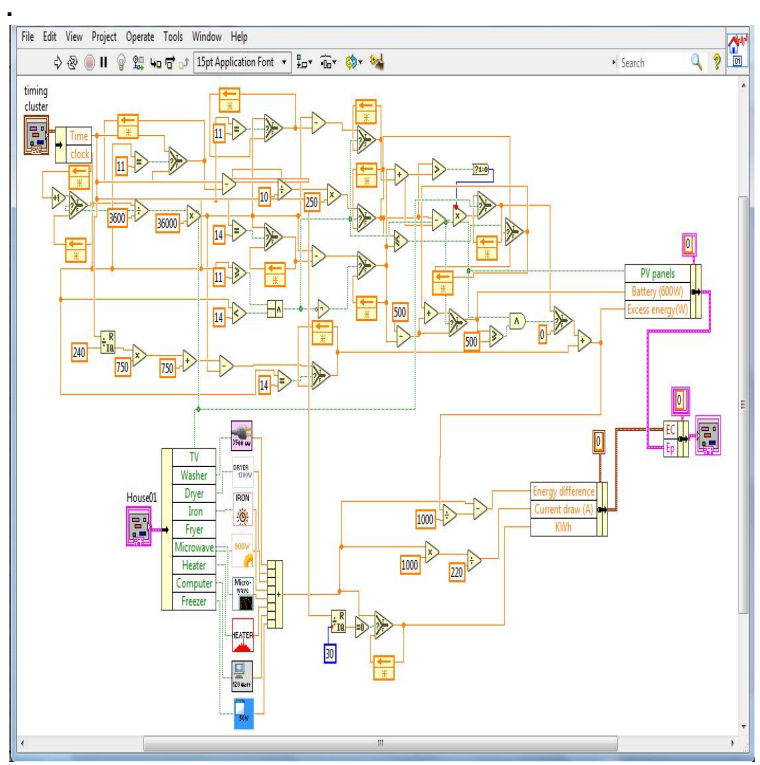

Fig. 6 House 01 block diagram.

Figure 6 shows the total block diagram of house 01 where both energy consumption and production included, that will be illustrated in details below . b- The Bidirectional (Two-Way Flow Of Electricity) meter reading : Measuring Customer Energy consumption:

- Block diagram A represents 8 house appliances, each with an on/off button where all are grouped in a cluster ,the Kwh consumed by the 8 appliances are added in order to calculate the totale Kwh consumed of the house.An example of microwave subVI is shown in Figure 8.

- Block diagram B: A cluster is used to represent all smart meter output: current draw, total Kwh with an update every30sec ,energy difference between Kwh and excess energy .

- Block diagram C: represents subVI of the microwave which is a function that calculate the Kwh consumed by counting the ON time of the microwave accumulatively, a conversion into hour is done then a multiplication with wattage of the microwave, and dividing by 1000 to get energy consumed in kilowatt. All of the other house appliances are of this same subVI but with the right wattage.

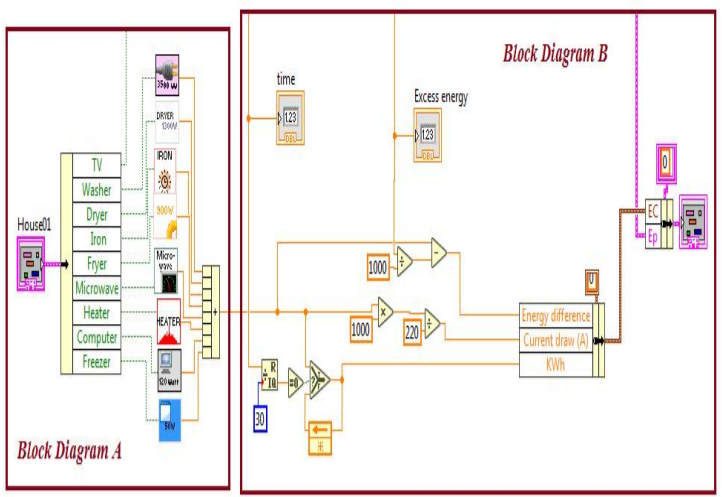

Fig. 7 Energy consumption Block diagram.

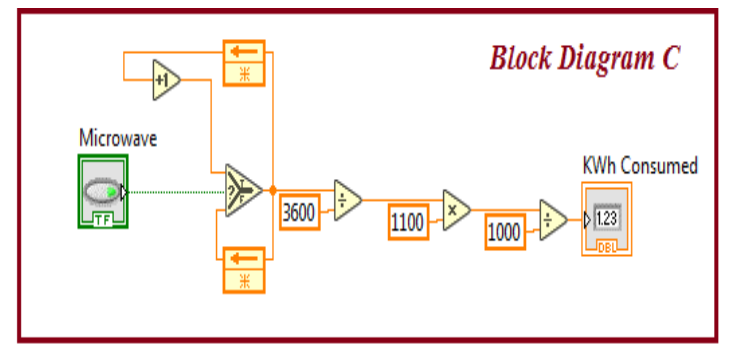

Fig. 8 Microwave subVI.

c- Measuring photovoltaic panels Energy production

\section{Photovoltaic solar panel (PV Panels):}

Photovoltaic (PV) technologies are an expanding market segment in modern power 
markets. Photovoltaic use large areas of photovoltaic cells, known as PV or solar cells, to directly convert sunlight into usable electricity. These cells are usually made from silicon alloys, solar panels energy depend on the size of the panel, the efficiency at which the panel converts sunlight to electricity, the amount of sunlight the panel receives, the orientation of the panel relative to the sun, and a host of other variables.

\section{Grid Fall Back:}

With a grid fallback system, the solar array generates power, which in turn charges a battery bank. Energy is taken from the battery and run through an inverter to power one or more circuits from the distribution panel in the house.

When the batteries run flat, the system automatically switches back to the grid power supply. The solar array then recharges the batteries and the system switches back to solar power.

With a grid fallback system, you do not sell electricity back to the electricity companies, until your batteries have been charged first. Once your batteries have been fully charged, any excess power generated will be exported to the grid.

Grid fallback systems provide most of the benefits of a grid interactive system, with the additional benefit that you use your own power when you need it, rather than when the sun is shining. This reduces your reliance on external electricity supplies during peak load periods, which ensures that your system has an overall environmental benefit.

Solar panels rarely power electrical equipment directly. This is because the amount of power the solar array collects varies depending on the strength of the sunlight. This makes the power source too variable for most electrical equipment to cope with [8].

d- Measuring the power of the PV Panels:

The requirements for solar electricity in the ten houses that are to run lighting or some relatively low-power electrical equipment such as a laptop computer, a small TV, a compact fridge or other appliances with small wattages is considered.

The solar panels generate electricity which is stored in battery bank. The PV module charges the battery bank during the 3hours of full sun starting at 11AM, where systems are designed to always have enough power to charge the battery bank. No excess energy will be fed into the grid unless the batteries are fully charged and the home appliance connected to the grid is turned off. When PV modules are off, the appliance will draw energy from the battery bank.

The battery banks installed are assumed to be enough for energy consumption when PV panels are off and the PV module is 250watt under standard test conditions.

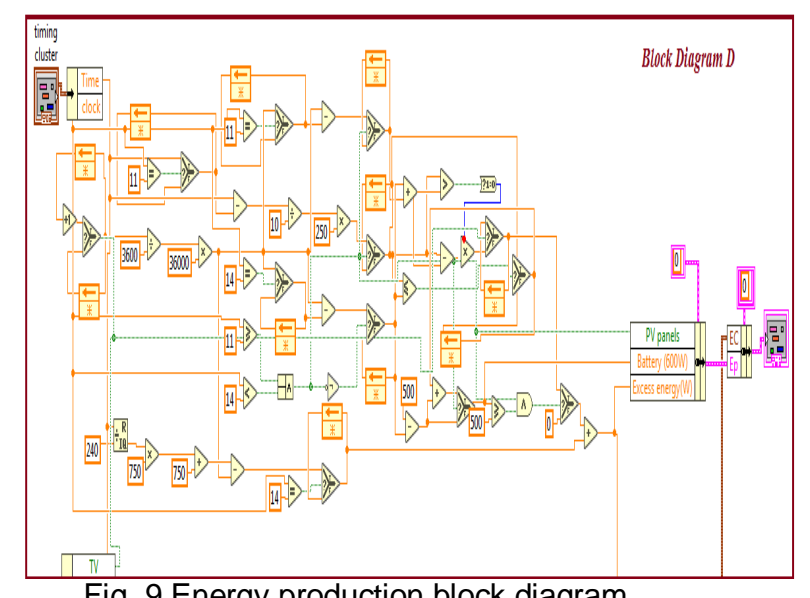

Fig. 9 Energy production block diagram.

- Block Diagram D in figure 9 represents a complex block diagram for energy production system where it performs the following functions :

$\checkmark$ Turns on a LED when the PV panels start producing energy at 11:00 till 14.00.

$\checkmark \quad$ When PV panels goes ON the batteries get charged immediately, after charging any excess of energy will be sent to the grid if the appliance connected to the PV module is off.

\section{The real time simulation}

\section{a- Load profile:}

The term load profile describes the pattern of electricity usage for a customer or a group of customer over a given period [9].

A load profile will vary according to customer type (typical examples include residential, commercial and industrial), temperature and holiday seasons. It enhances our knowledge of electrical consumption patterns. Besides, it has many applications including demand response, load forecasting, and non-technical loss detecting, etc.

With widespread use of $\mathrm{AMI}$ equipment, a lot of information can be mined from electrical consumption data of individual customers through load profiling [9].

Using waveform chart we draw the load profile of the ten houses for duration of 3days as shown in figure 10 . 
Figure 10 represents the accumulating $\mathrm{kWh}$ consumed by the ten houses.

Figure 11 represents the instantaneous $\mathrm{kWh}$ consumed by the ten houses the energy provided by the utility (we took $10 \mathrm{kw}$ as an example) and the limit of energy provided from the utility plus the Solar Panels (12kw). If the limit of $12 \mathrm{Kw}$ is exceeded some loads would be turned off automatically this is known as direct load control as we will see in the next title.

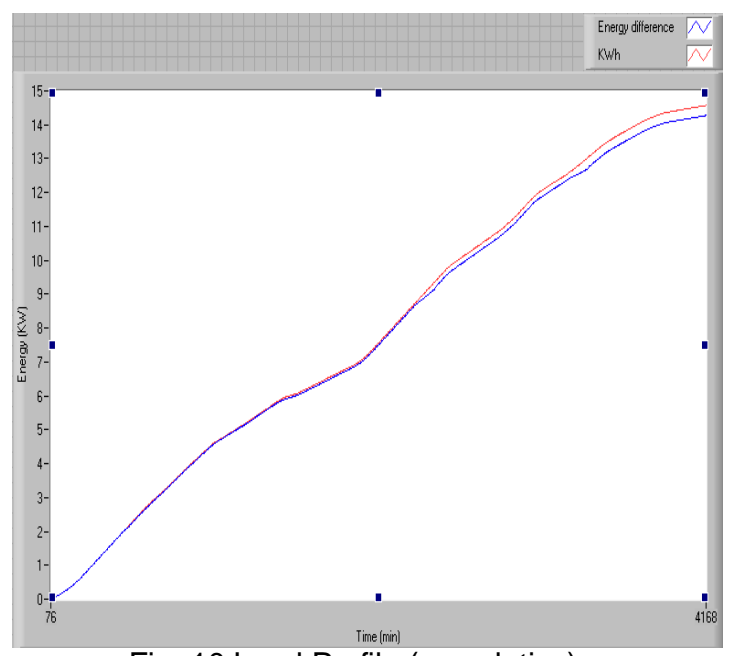

Fig. 10 Load Profile (cumulative).

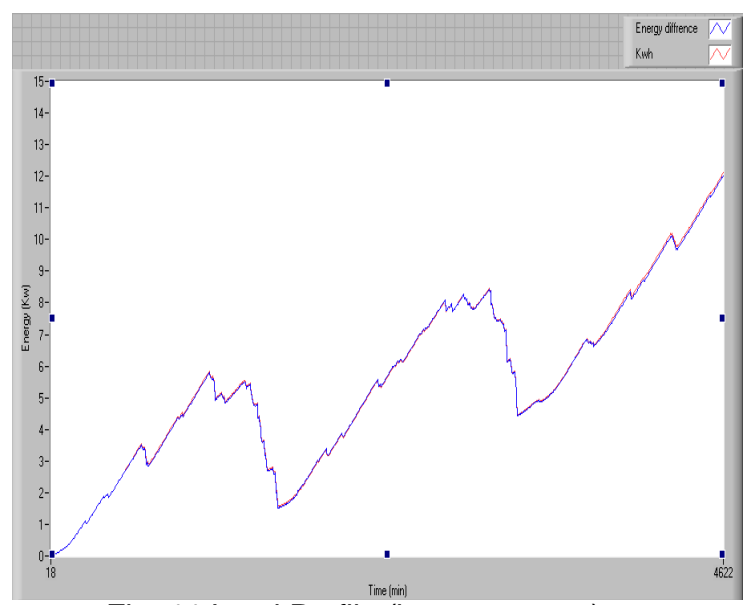

Fig. 11 Load Profile (instantaneous).

For instance, taking only energy consumption, the customers will benefit from metering solutions through greater understanding of their own energy consumption, allowing them to better manage costs of their usage. It would also allow them to understand which energy plan may suit their consumption more effectively. Suppliers benefit by meeting energy demand, improving efficiencies in load management and smoothing out demand by offering customers more tailored tariff plans.

In general, by load profiling everyone can benefit by better understanding and managing energy demand. Additionally, a potential value added maybe also achieved by forecasting future demand to reduce the strain on the system during heavy usage periods.

\section{b- Direct Load control}

Load control system can be operated by an electricity supplier or network operator, a market or system operator, a demand side response service provider or end user. Special tariff rates such as real time pricing, day ahead pricing, time of use pricing, and critical peak pricing should be introduced with load control, aimed at compensating the reduced autonomy of the consumer. This allows the operator the right to control load demand. Where modern smart metering systems support information display devices, appliance and equipment controllers, and integrated load control systems. Smart appliances individually measure the energy consumption of each appliance at the consumer end. The data from these devices are collected at the control centre in near real time. The control signals are sent back to the appliance controllers from the control centre. Therefore two-way communication link should be established to communicate between the load manager and the controlled loads.

At the operating centre, the load manager sends signals to initiate or terminate a load control program. The signal may also be generated automatically when the demand exceeds the pre-set load levels. Instructions are given to reduce the demand to pre-set levels, cycle the loads on and off in regular intervals, or to switch off the load completely. [7]

In our work we proposed a LABVIEW program where the consumer will be notified by the excess of energy that he is using (practically, this could be done by sending him a text message to his mobile phone) and ask him to turn off the loads that consumes a big amount of electricity such as an air conditioner after having them turned off automatically by the utility for a short period of time.

Figure 12 illustrates a dialogue box that will pump out to alert the user and stop the 
execution of the program until clicking on the Ok button and turning off the loads.

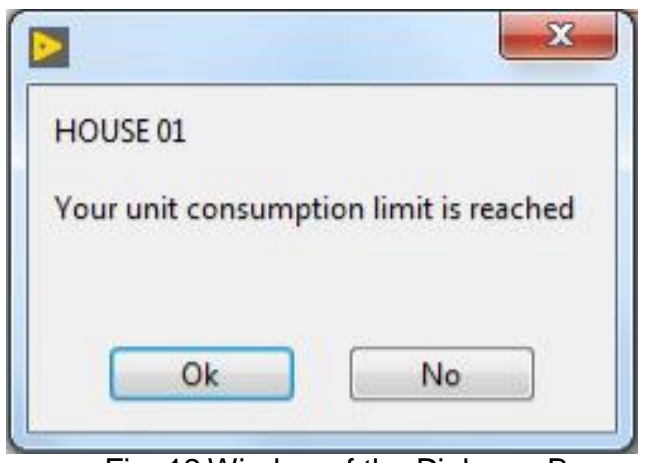

Fig. 12 Window of the Dialogue Box.

Figure 13 shows the Block diagram of the dialogue box, where it is connected to the microwave and iron.

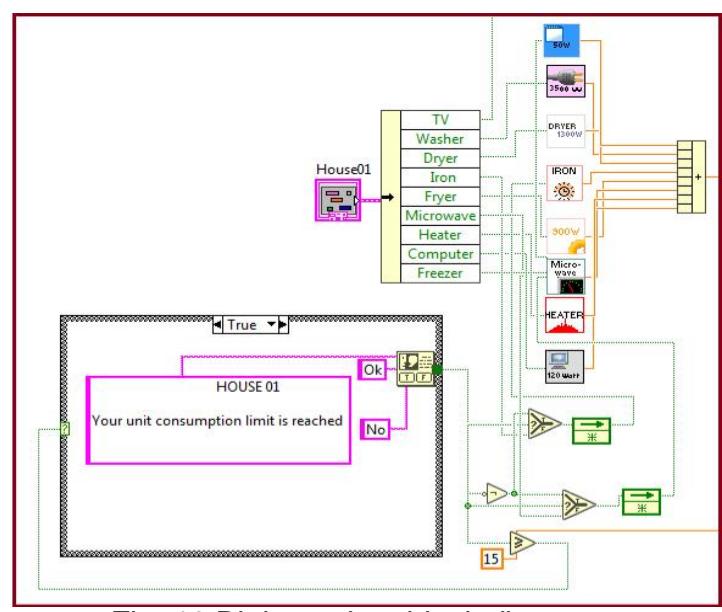

Fig. 13 Dialogue box block diagram

Figure 14 shows the front panel of the dialogue with limit of $15 \mathrm{KWh}$.

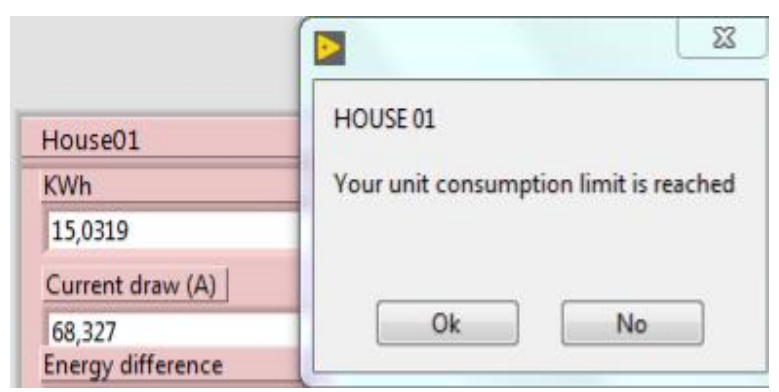

Fig. 14 Dialogue Box front panel.

\section{The Centralised Management And control system}

The Control Center (CC) or Data Management System (DMS) is in charge of receiving and storing the metering data for processing purposes. The $\mathrm{CC}$ can be seen as a modular system formed by the Meter Data Management System (MDMS), which manages the metering data and additional secondary modules in charge of end-users applications, weather forecasting systems, geographical information systems, control applications, and load management.

Figure 15 represents the control centre front panel where the data and parameters of ten houses are displayed.

Figure 16 represents the control centre block diagram.

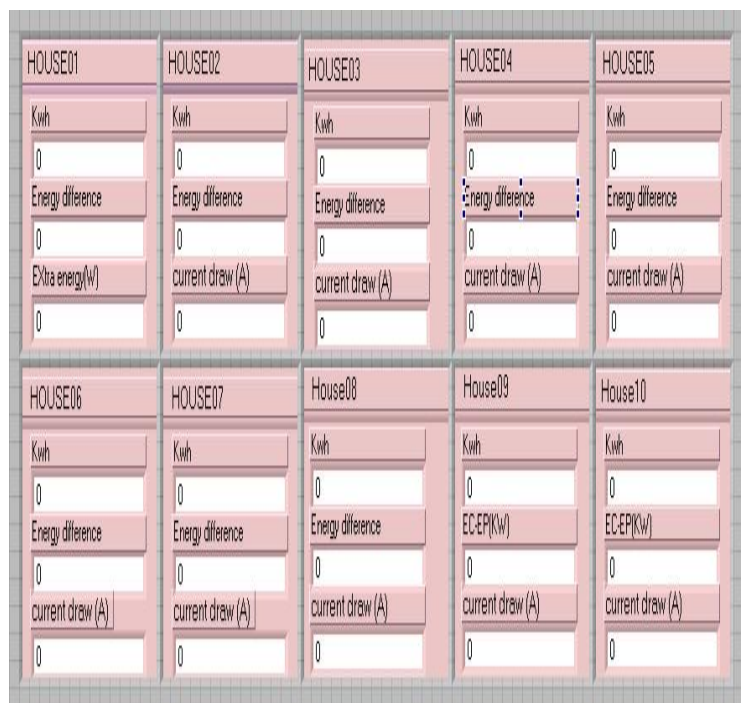

Fig. 15 Control Center front panel

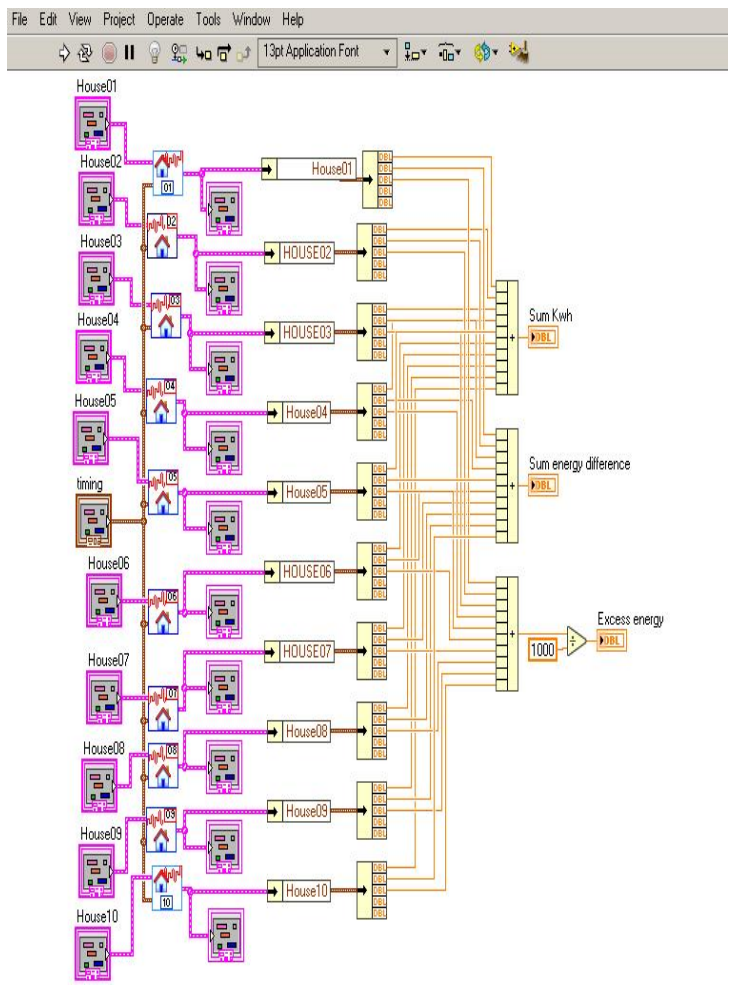

Fig. 16 Control center block Diagram. 


\section{PRICING ESTABMISHEMENT}

BILL

This section focuses on the importance of the net metering and adopting correct power pricing policies to maximize the net economic benefits of electricity consumption to society. An example of how net metering works, is illustrated by the simulation of Three scenarios using LABVIEW software that shows the different cases of homes energy consumption that may occur and its associated billing system description. At the end of a given month, the customer is billed only for the net electricity used. If more energy is produced than consumed, producers receive benefit for this negative balance, such as, renewable energy credits (REC), which is credited on the customer's account toward the next billing cycle. If at the end of the year a surplus remains, then the customer depending on the utility policy may (a) be paid for the total REC collected at avoidance cost rate or retail cost rate, (b) the total REC collected can be transferred and could be used as a compensation for a possible negative balance in the following years, or (c) the total REC collected are granted back to the utility [5].

We will also go into details about the different form of pricing policies that exists since they are a valuable "soft" tool for managing demand and reducing electricity consumption and peak demand [10].

\section{A. Net Metering:}

Net metering is a new concept where an instrument which has a special metering and billing agreement between utilities and their customers, facilitates the connection of small, renewable energy-generating systems to the power grid. This new program is being developed to encourage small scale renewable energy systems to ensure that customers always have a reliable source of energy even when their renewable generators are not producing energy, and to provide substantial benefits to the electric power-generating system as well as the environment. When a net metering client's renewable generator is producing more power than is being consumed, the electric meter runs backward generating credits. Whenever the net metering customer uses more power than is being produced, the meter runs forward normally. Net metering customers are charged only for the net power that they consume from the electricity service provider that has accumulated over a specific period. In other words, if their renewable energy generating systems make more electricity than is consumed, they may be credited or paid for the excess electricity contributed to the grid over that same period. Net metering is also a way to increase the energy in the power grid to keep up with increase in demand during peak power use times, and this is of particular interest to states facing power shortages [8].

\section{B. Final Billing}

The power produced by the solar system is consumed by the house, and the surplus power is sent to the Grid. We have designed an Energy Billing Statement with details included for explaining how the statement is calculated. Three examples of how the statement may appear are shown below; the first is an example of a normal solar billing statement (Illustration A). The second is an example of a solar billing statement for someone whose solar system produced more energy than they received from the electrical grid, resulting in an Excess Generation credit being applied to their account (Illustration B). The last one is very similar to the second one but this time the consumer has been absent for a long period, thus resulting in a considerable excess generation credit (Illustration C).

\section{Illustration A}

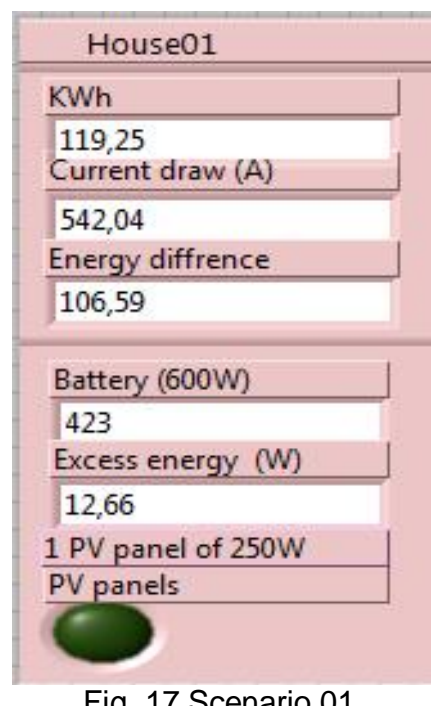

We extract the required information to calculate our bill from Figure 3-1 and proceeded as follow: 


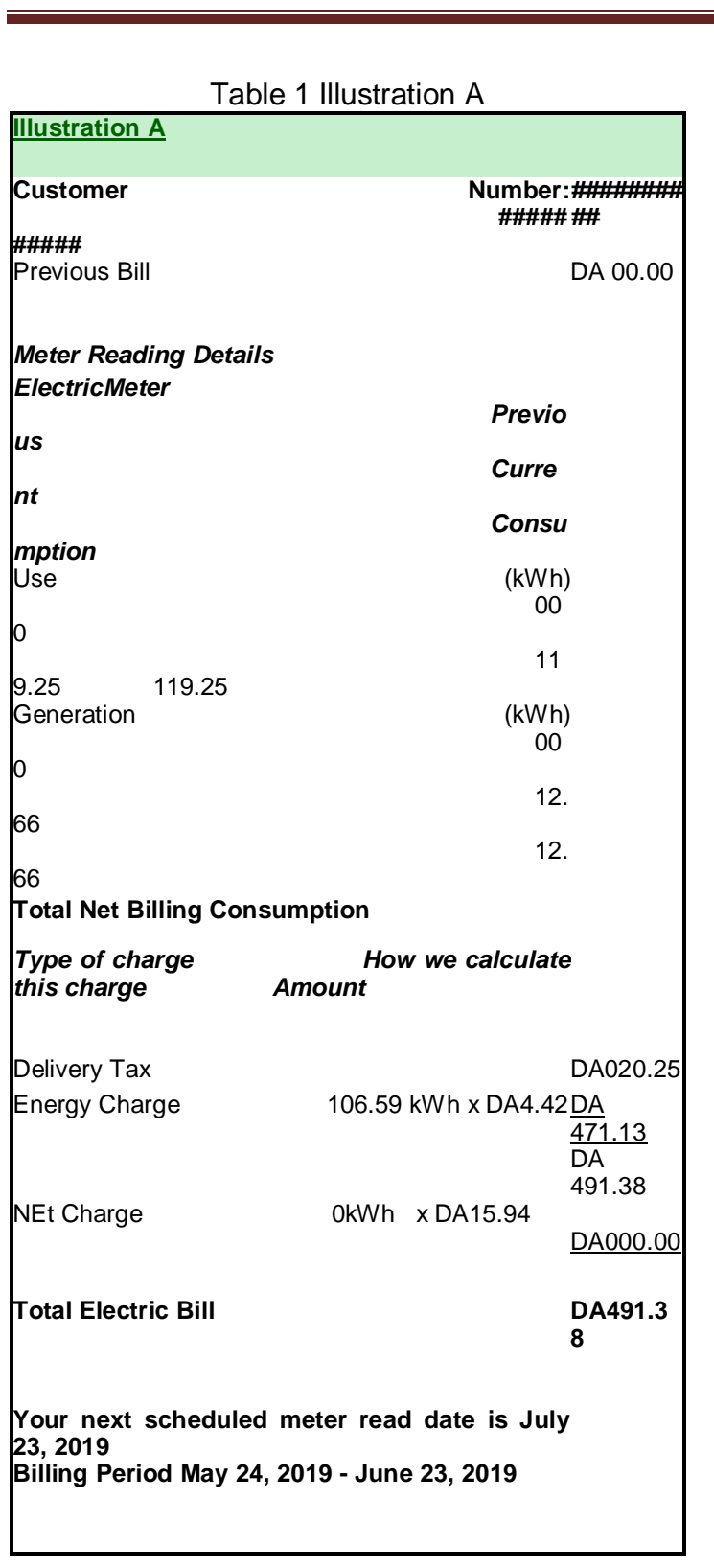

\section{Illustration B}

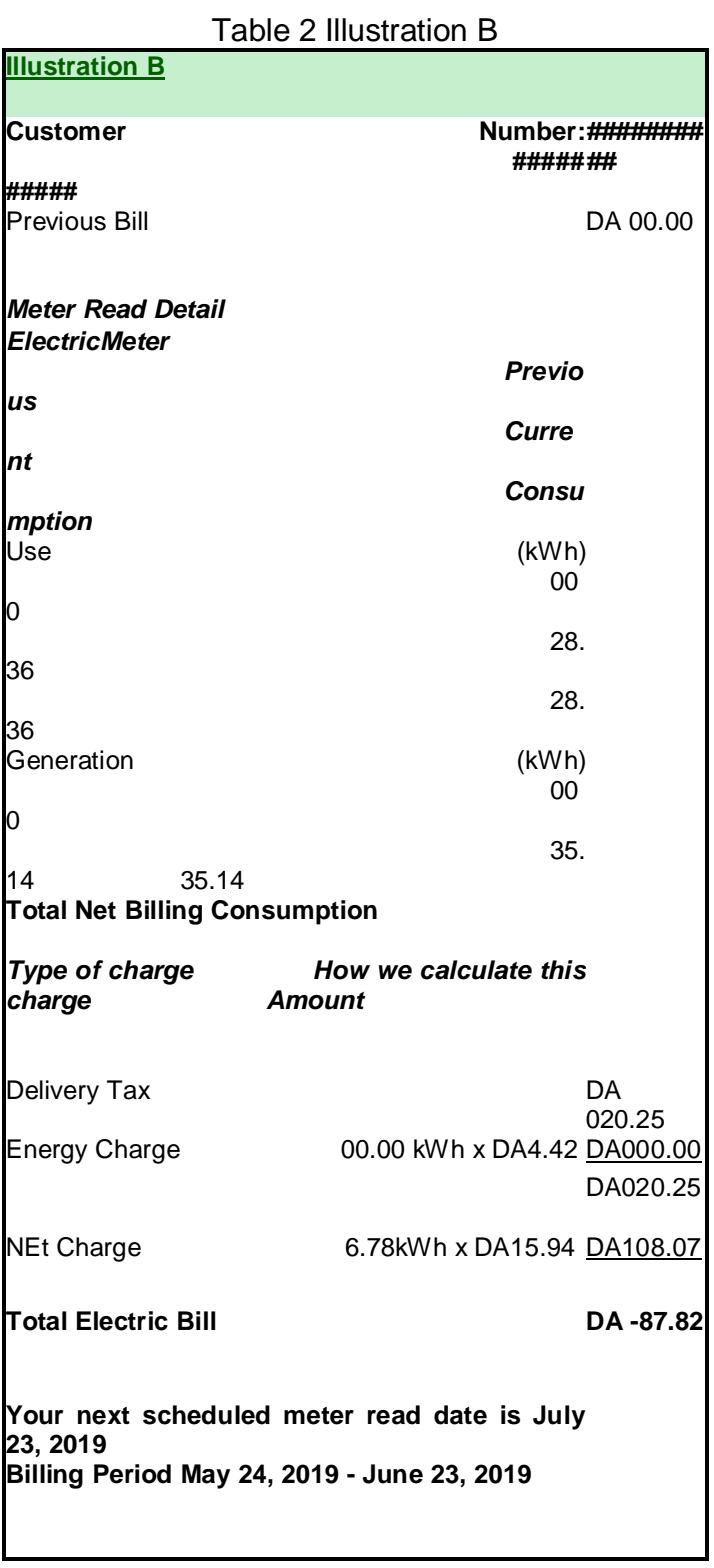

\section{Illustration C}

\begin{tabular}{|l|}
\hline \multicolumn{1}{|c|}{ House 4} \\
\hline KWh \\
\hline 28,36 \\
current draw (A) \\
\hline 128,91 \\
\hline Energy difference \\
\hline$-6,78$ \\
\hline Battery (2000W) \\
\hline 1564 \\
\hline Excess energy(W) \\
\hline 35,14 \\
\hline PPV panels of 250W \\
\hline PV is ON \\
\hline
\end{tabular}

Fig. 18 Scenario 02

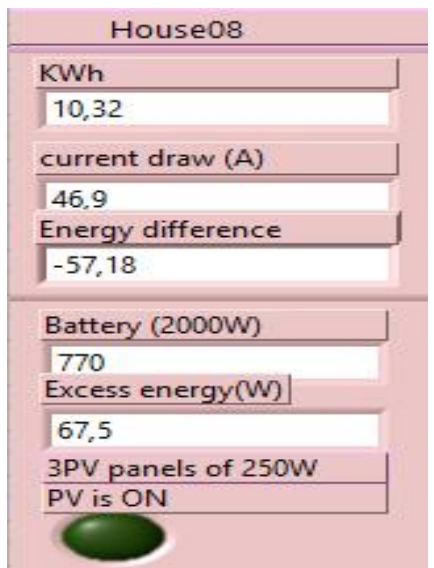

Fig. 19 Scenario 03 


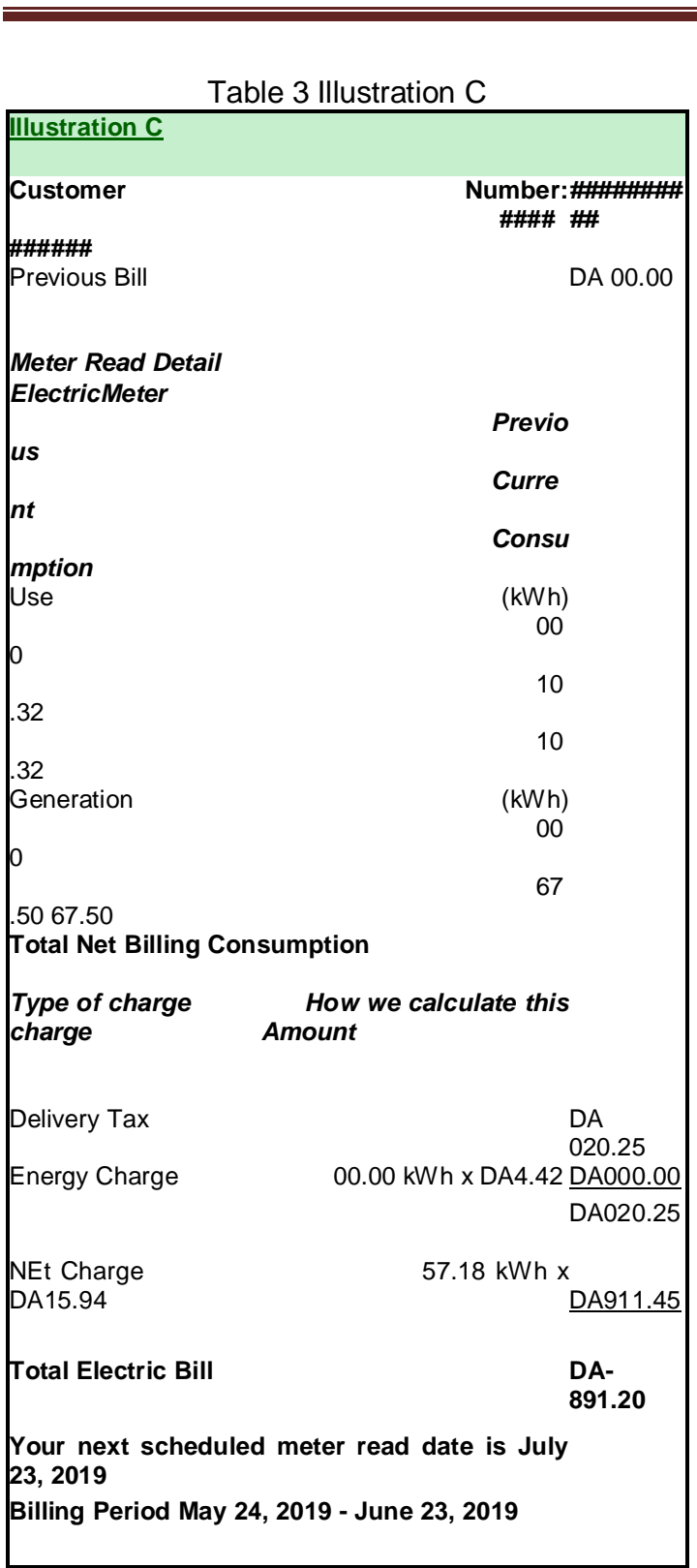

\section{Pricing Policy}

The Distribution companies are starting to adopt a new pricing strategy also known as Load management and demand control in order to reduce the peak demand, and save the electricity cost. When it comes to electricity savings the consumers can change the consumption patterns under different tariff schemes.

Modern smart metering systems support following tariff schemes

- Real time pricing (RTP):

Price signals are generated in hourly basis or half hourly basis, in most cases reflecting the true cost of electricity. Consumers are updated in regular intervals (hourly of half hourly) by sending price signals to the smart meters or home display units.
- $\quad$ Time of use pricing (TOU):

It utilizes time-varying electricity pricing signals to stimulate customers to shift their power consumption from peak hours to offpeak hours, which eventually reduces the peak load of the grid. By setting high electricity prices in peak-demand hours and low prices in off-peak-demand hours, the user is encouraged to shift the use of some appliances such as washing machines to low price hours, to save the household's electricity cost. The most widely adopted time-varying electricity tariff is Time-of-Use (TOU) which is widely employed in many countries. In TOU, one day is divided into multiple periods and different electricity prices are applied in each period.

- Critical peak pricing:

The tariff is same as the TOU but higher rates are applied at higher demands. Prior to applying the higher rates, the consumers are notified.

- Day ahead pricing:

This is similar to RTP, but the price schedule is sent to the consumer $24 \mathrm{~h}$ before the rates are applied. Generally, different prices are defined for different time locations (on hourly basis most of the time) of the next day [7].

\section{CONCLUSION}

Electric power is a crucial form of energy in the world today. A well maintained electric network is required to provide accuracy, reliability and sufficiency to consumers. The current electric infrastructure is aging and it is being pushed to do more than it was originally designed to do. Modernizing the grid to make it "smarter" and more resilient through the use of technologies that communicate and work together to deliver electricity efficiently and allow the consumers to better manage their own energy consumption and costs because they have easier access to their data.

\section{Acknowledgments}

This work is part of the research activities within the Laboratory of Signals and Systems, university of Boumerdes, Algeria. Special thanks to Professor Hamid BENTARZI, the director of the signals and systems Laboratory at the University of Boumerdes-Algeria for his valuable advice and continual support. 


\section{REFERENCES}

[1]- N. Uribe-Pérez et. al., "State of the Art and Trends Review of Smart Metering in Electricity Grids", Applied Sciences, MDPI, 6(3), 68, 2016. https://doi.org/10.3390/app6030068.

[2] S. Massoud Amin and B.F. Wollenberg, "Towards a SmartGrid: Power delivery for the $21^{\text {st }}$ Century, 'IEEE Power \& Energy Magazine, Vol. 3, No.5 pp 34-41 Sep-Oct 2005.

[3] TrongNghia Le, Wen-Long Chin, DangKhoa Truong and Tran Hiep Nguyen, "Advanced Metering Infrastructure Based on Smart Meters in Smart Grid", Book chapter, INTECH open, 2016. DOI:10.5772/63631.

[4] P. Koponen et. al., "Definition of Smart Metering and Applications and Identification of Benefits" VTT Technical Research Centre of Finland. 2008.

[5] S. Palaniappan et. al., " Home Automation Systems - A Study", International Journal of Computer Applications, Vol. 116 - No. 11, April 2015.

[6]- Sharda R. Katre and Dinesh V. Rojatkar, "HOME AUTOMATION: PAST, PRESENT AND FUTURE", International Research Journal of Engineering and Technology (IRJET), Vol.4, issue 10, 2017.

[7] K. S. K. Weranga, Sisil Kumarawadu and D. P. Chandima, "Smart Metering Design and Applications", Springer Singapore, 2014.

[8] Andreas Poullikkas, George Kourtis, loannis Hadjipaschalis, "A review of net metering mechanism for ectricity renewable energy sources", INTERNATIONAL JOURNAL OF ENERGY AND ENVIRONMENT, Volume 4, Issue 6, 2013 pp.975-1002.

[9] K. L. Lo, Z. ZAKARIA, M. H. SOHOD, "Determination of Consumers' Load Profiles based on Two-stage Fuzzy C-Means", Proceedings of the 5th WSEAS Int. Conf. on Power Systems and Electromagnetic Compatibility, Corfu, Greece, August 23-25, 2005. Pp.212-217.

[10] Mohan Munasinghe and Jeremy J. Warfor, "Electricity Pricing Theory and Case Studies", The Johns Hopkins University Press., 1982. .

[11] A. B. M. Shawkat Ali, "Smart Grid: Opportunities, Developments, and Trends", Springer, London, 2013.

[12] Gabriele Lobaccaro, Salvatore Carlucci and Erica Löfström, "A Review of Systems and Technologies for Smart Homes and Smart Grids", Energies, 2016, 9(5), 348; https://doi.org/10.3390/en9050348.

[13] Ganiyu A. Ajenikoko, Anthony A. Olaomi, "Hardware Design of a Smart Meter", Int. Journal of Engineering Research and Applications, Vol. 4, Issue 9( Version 6), September 2014, pp.115-119.

[14] S. Zahurula et. al., "ELECTRICITY MEASUREMENT SENSOR: A REVIEW ON
APPLICATION TO SMART METER COMMUNICATION", International Conference on Engineering Education 2013 Madinah, Kingdom of Saudi Arabia, 22-25 December 2013.

[15]- Ezeodili Echezona Ugonna, Adebo King Ademola, AkinbulireTolulope Olusegun, "Design and construction of a smart electric metering system for smart grid applications: Nigeria as a case study", International Journal of Scientific \& Engineering Research Volume 9, Issue 7, July-2018. 